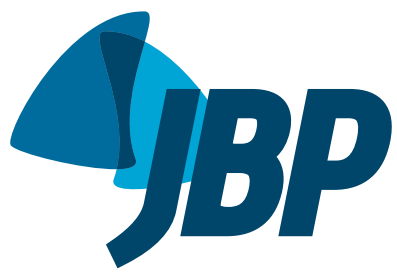

\title{
Idiopathic pleuroparenchymal fibroelastosis: incidental findings in a patient with suspected pneumonia
}

\author{
Gaetano Rea1, Venerino Poletti ${ }^{2}$, Carlo ladevaia ${ }^{3}$, Marialuisa Bocchino ${ }^{4}$, \\ Gennaro Mazzarella ${ }^{3}$
}

\section{TO THE EDITOR:}

Idiopathic pleuroparenchymal fibroelastosis (IPPFE) is an uncommon disease of the pleura and lungs. In 2013, IPPFE was classified as a rare interstitial lung disease (ILD) in a joint American Thoracic Society/European Respiratory Society statement on the classification of the idiopathic interstitial pneumonias. ${ }^{(1)}$ Characterized by fibrosis, together with thickening of the pleural and subpleural tissue (subpleural fibroelastosis), IPPFE predominantly affects the periphery of the upper lung lobes, with volume loss, as first described by Frankel et al. in 2004.(2) To date, no more than forty cases of IPPFE have been described in the literature in English. There is currently no consensus regarding the diagnostic criteria used in order to classify IPPFE, as well as regarding whether or not it represents a real, new, specific entity. Although the etiology is unknown, conditions associated with the disease include infections, bone marrow transplantation, autoimmunity, and possibly genetic predisposition. Clinically, patients with IPPFE usually present with chronic respiratory symptoms such as dyspnea and dry cough. The diagnosis of IPPFE is established on the basis of clinical, radiological, and histopathological findings. Chest HRCT scans show marked subpleural consolidations with irregular thickening; distortion, usually located in upper lung lobes ("pleural cap"); subpleural bronchiolectasis; reticular opacities; and, in some cases, mild honeycombing. These features are similar to those of other forms of idiopathic pulmonary fibrosis. However, in IPPFE, they are seen mainly in upper lobes, being rare or absent in the lower lobes. ${ }^{(3,4)}$ The histological characteristics of IPPFE are homogeneous subpleural fibrosis and abundant elastic fibers on elastic fiber stains. ${ }^{(5)}$

A 55-year-old nonsmoking female presented to her pulmonologist with fever $\left(38^{\circ} \mathrm{C}\right.$ for six days), worsening of dyspnea under stress, and a productive cough. The patient stated that she had not been previously exposed to environmental allergens or asbestos. She further stated that she had not used any drugs and had not been previously exposed to environmental allergens, as well as that she had not been under treatment with chemotherapy or radiotherapy. She tested positive only for autoantibodies against endomysium; other serological tests were negative. Physical examination showed that, over a period of six months, she had lost weight ( $5 \mathrm{~kg}$ ) and muscle mass. There was also a reduction in oxygen saturation $\left(\mathrm{SaO}_{2}\right.$ of $81 \%$ on room air). On auscultation, there were reduced breath sounds and mild bilateral rales in the upper lung fields. Heart rate and rhythm were normal. Her extremities were also normal, without digital clubbing. Pulmonary function tests showed normal FVC (96\%) and normal FEV $_{1}(91 \%)$, although the DLCO was reduced (61\%). An initial chest X-ray (not shown) revealed marked, irregular opacities in the subpleural region of the upper fields, with mild upper hilar retraction. Fiberoptic bronchoscopy with BAL was carried out, and neutrophils were found to account for $72 \%$ of the inflammatory cells (normal value, $1-2 \%$ ). Microbiology tests were negative. To elucidate the chest $\mathrm{X}$-ray and BAL findings, we performed chest HRCT. The HRCT scans showed peripheral foci of lung consolidation, with distortion and irregular pleuroparenchymal thickening, that was most evident in the subpleural regions of the upper lobes, accompanied by bronchiolectasis, mild interlobular septal thickening, and (in rare cases) microcystic subpleural changes (Figure $1 \mathrm{~A}$ ). Scans of the lower lung fields (middle lobe and lingula) showed considerably fewer fibrotic changes, no distortion or other interstitial disease being observed in the apical and basal segments of the lower lobes (Figures $1 \mathrm{~B}$ and $1 \mathrm{C}$, respectively). These HRCT characteristics pose a diagnostic dilemma for the radiologist, who must make the differential diagnosis among IPPFE, sarcoidosis, and (albeit less likely) chronic hypersensitivity pneumonia. Therefore, we also performed a pleuroparenchymal frozen section biopsy in the upper lobes. Histopathological findings from elastic fiber staining revealed distortion and marked pleural thickening, with evidence of dense fibroelastotic tissue in the pleura and alveolar walls, together with sparse fibroblastic foci near the transition from the lung to the pleura (Figure 1D). On the basis of the clinical and radiological data, a diagnosis of IPPFE was made. In conclusion, although there are specific HRCT features that are suggestive of IPPFE, the disease continues to be underdiagnosed, probably because there is still a significant lack of knowledge and awareness of this entity. A diagnosis of IPPFE should be considered in cases of pulmonary fibrosis that is located predominantly in the upper lobes. The differential diagnosis includes advanced hypersensitivity pneumonia, advanced sarcoidosis, advanced smoking-related ILD, asbestosis, connective tissue disease, radiation pneumonia, and drug-induced lung disease. The possibility of IPPFE should 

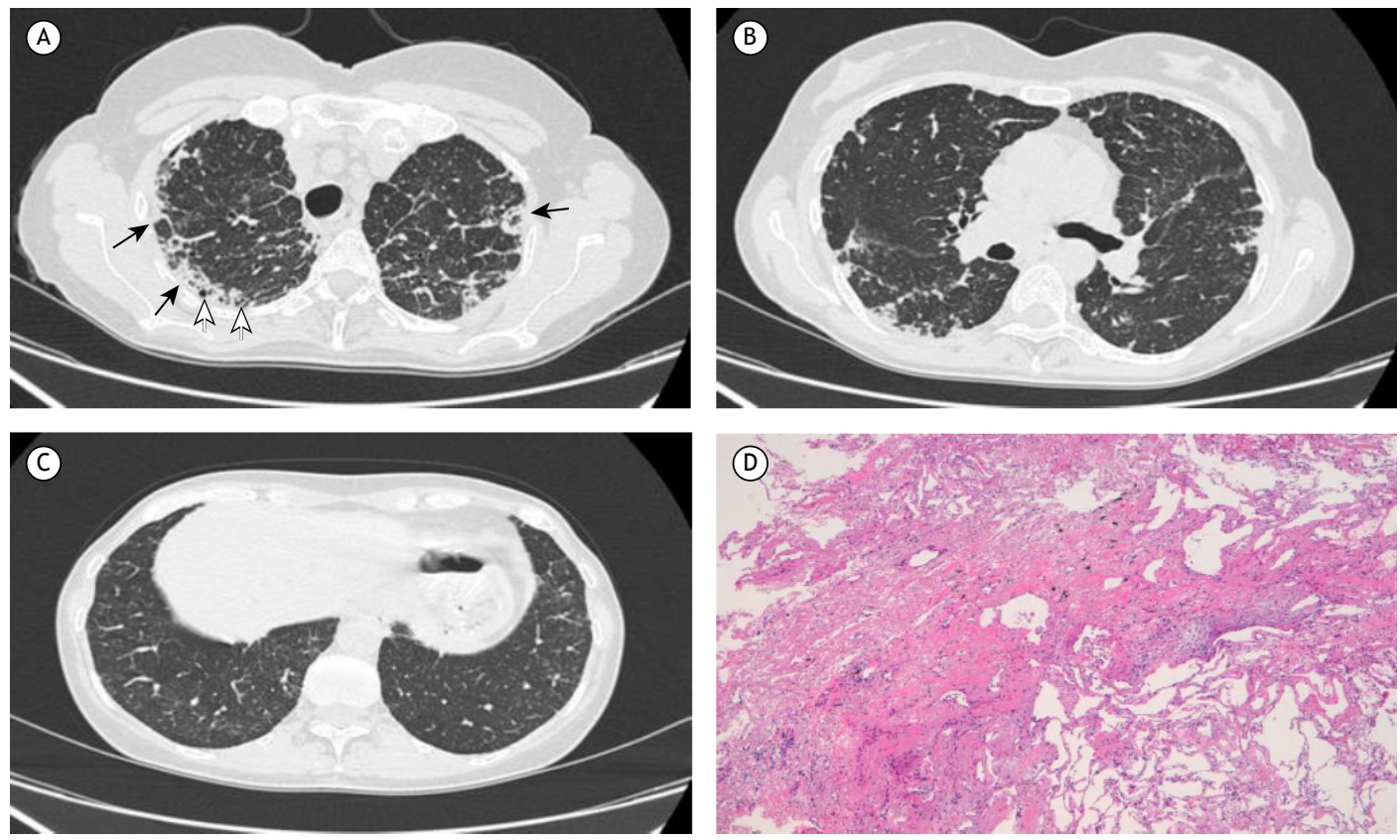

Figure 1. Chest HRCT and histology: A) HRCT scan showing marked subpleural thickening and irregular consolidations (black arrows), reticular opacities in the lung parenchyma at upper lobes, microcystic subpleural changes (white arrows), and bronchiolectasis; B) HRCT scan showing sparse fibrotic subpleural changes in the apical segments of the lower lung lobes, most pronounced on the right; C) HRCT scan showing near absence of fibrotic changes in the basal segments of the lower lung lobes; and D) histological section showing dense subpleural fibroelastotic tissue with an abrupt transition from pathological to normal lung parenchyma, containing a fibroblastic focus.

therefore be borne in mind when the radiological evidence is not consistent with other, more well-defined ILDs. It is recommended that surgical biopsy (using video-assisted thoracoscopic surgery) - or better yet frozen section biopsy-be performed in the affected area. A greater understanding of the etiology, risk factors, prognosis, and therapy related to IPPFE is needed in order to manage this disease in the future.

\section{REFERENCES}

1. Travis WD, Costabel U, Hansell DM, King TE Jr, Lynch DA, Nicholson $A G$, et al. An official American Thoracic Society/European Respiratory Society statement: Update of the international multidisciplinary classification of the idiopathic interstitial pneumonias. Am J Respir Crit Care Med. 2013;188(6):733-48. http://dx.doi.org/10.1164/ rccm.201308-1483ST

2. Frankel SK, Cool CD, Lynch DA, Brown KK. Idiopathic pleuroparenchymal fibroelastosis: description of a novel clinicopathologic entity. Chest. 2004;126(6):2007-13. http://dx.doi. org/10.1378/chest.126.6.2007

3. Piciucchi S, Tomassetti S, Casoni G, Sverzellati N, Carloni A, Dubini
A, et al. High resolution CT and histological findings in idiopathic pleuroparenchymal fibroelastosis: features and differential diagnosis. Respir Res. 2011;12:111. http://dx.doi.org/10.1186/1465-9921-12 111

4. Camus P, von der Thüsen J, Hansell DM, Colby TV. Pleuroparenchymal fibroelastosis: one more walk on the wild side of drugs? Eur Respir J. 2014;44(2):289-96. http://dx.doi.org/10.1183/09031936.00088414

5. Becker CD, Gil J, Padilla ML. Idiopathic pleuroparenchymal fibroelastosis: an unrecognized or misdiagnosed entity? Mod Pathol. 2008;21(6):784-7. http://dx.doi.org/10.1038/modpathol.2008.56 